\title{
Uma Parceria Público-Privada: Influência de um Programa de Atividade Física em Índices de Saúde
}

\author{
A Public-Private Partnership: Influence of a Physical Activity \\ Program in Health Indicators
}

\author{
VALÉRIA CRISTINADE FARIA ${ }^{1}$ \\ GUILHERME PEREIRA OLIVEIRA ${ }^{2}$ \\ THAYNARA NATHALIE CARVALHO ESTEVAM ${ }^{2}$ \\ ALBENÁ NUNES DA SILVA ${ }^{3}$ \\ AFONSO TIMÃO SIMPLÍCIO ${ }^{4}$ \\ GUILHERME DE AZAMBUJAPUSSIELDI ${ }^{5}$
}

\section{RESUMO}

Introdução: A prática regular de atividade física traz benefícios tanto na promoção de saúde quanto na prevenção e combate de doenças, o que se torna importante dentro de um ambiente de trabalho e na perspectiva de um plano de saúde. Objetivo: Avaliar a eficácia de um programa de intervenção com atividade física sobre os seguintes parâmetros de saúde: índice de massa corporal (IMC), índice de cintura quadril (ICQ), percentual de gordura corporal (\%GC), percentual de massa magra (\%MM), e perfil de estado de humor. Material e Métodos: Foram utilizados como instrumentos de avaliação o protocolo de dobras cutâneas de Faulkner e a Escala de Humor de Brunel. Trata-se de uma intervenção com duração de seis meses e frequência mínima de seis vezes ao mês, em 44 participantes do programa de atividade física e saúde, oferecido aos servidores públicos da UFV- Campus Florestal que possuem o plano de saúde Agros e seus dependentes. $\mathrm{Na}$ análise estatística foi utilizado o teste t pareado e um $\mathrm{p} \mathrm{d"}$ 0,05. Resultados: Os resultados significativos foram: diminuição e aumento do \%GC e \%MM, respectivamente, para todos os grupos atendidos; diminuição no ICQ para o grupo de servidores aposentados ( $p=0,032)$; diminuição da raiva para o grupo de servidores ativos $(p=0,044)$ e aposentados $(p=0,024)$; e aumento do vigor para os dependentes $(p=0,003)$. Conclusão: Diante da melhora dos parâmetros avaliados a intervenção se mostrou benéfica à saúde dos participantes, sendo positivo para o plano de saúde e para o trabalho.

\section{DESCRITORES}

Atividade Física. Intervenção. Saúde.

\begin{abstract}
Introduction: Regular physical activity provides benefits both in health promotion as in preventing and combating disease, which becomes important in a work environment and the perspective of a health plan. Objective: Was to evaluate the effectiveness of an intervention program with physical activity on following health parameters: body mass index (BMI), waist-hip ratio (WHR), body fat percentage (\%BF), percentage of lean mass (\% LM), and mood state profile. Material and Methods: Were used as assessment tools Faulkner skinfold protocol and the Brunel Mood Scale. This is an intervention lasting six months and with a minimum frequency of six times a month, in 44 participants of the physical activity and health program offered to civil servants of UFV- Campus Florestal that have the health plan Agros and their dependents. Statistical analysis was performed using paired t-test and a $\mathrm{p}$ d" 0.05 . Results: The significant results were: decrease and increase $\% \mathrm{BF}$ and\% $\mathrm{MM}$, respectively, for all treated groups; decrease in WHR for the group of retired employees $(p=0.032)$; decrease in anger for the group of active $(p=0.044)$ and retired employees $(p$ $=0.024)$; and increased force for dependents $(p=0.003)$. Conclusion: Given the improvement of the parameters evaluated the intervention proved to be beneficial to the health of the participants, thus positive for the health plan and for the work.
\end{abstract}

\section{DESCRIPTORES}

Physical activity. Intervention. Health.

\footnotetext{
Graduada em Educação Física. Mestre em Educação Física. Educadora Física do Agros. Instituto UFV de Seguridade Social. Gerência de Saúde. Setor Pró-Saúde - Florestal, Minas Gerais, Brasil.

2 Estudante de Educação Física da Universidade Federal de Viçosa/ Campus Florestal, Florestal, Minas Gerais, Brasil.

3 Professor Doutor Adjunto do Curso de Educação Física da Universidade Federal de Ouro Preto, Minas Gerais, Brasil.

4 Professor Mestre do Curso de Educação Física da Universidade Federal de Viçosa/ Campus Florestal, Florestal, Minas Gerais, Brasil.

5 Professor Doutor do Curso de Educação Física da Universidade Federal de Viçosa - Campus Florestal - Florestal - MG - Brasil.
} 
$\mathrm{A}$ saúde é composta de fatores que se relacionam à qualidade de vida ${ }^{1}$. A Organização Mundial de Saúde a define como um completo bem estar físico, mental, social, espiritual e não somente ausência de afecções e ades ${ }^{2}$. Assim, as estratégias e ações de promoção da saúde envolvem diversos ambientes e segmentos através de políticas públicas e de condições favoráveis ao seu desenvolvimento ${ }^{1}$.

No que se refere à atividade física, a Sociedade Brasileira de Medicina do Esporte aponta que a saúde e a qualidade de vida do homem podem ser preservadas e aprimoradas pela prática regular da mesma, além de contribuir tanto na prevenção quanto no combate de doenças crônicas não transmissíveis (DCNTs) ${ }^{3}$. Portanto, diminuir a inatividade física da população pode melhorar substancialmente a saúde da população mundial ${ }^{4}$.

As agências mundiais de saúde têm editado diversos documentos contendo estratégias globais para o incentivo às práticas saudáveis, sendo a alimentação e a atividade física regular constantemente recomendadas $^{5}$; um exemplo é a Política Nacional de Promoção da Saúde ${ }^{6}$, e mais recentemente, os programas Academia da Saúde integram as políticas de promoção de saúde no Brasil ${ }^{7}$.

No âmbito privado, em cumprimento da Lei 9.961/ 00, a Agência Nacional de Saúde (ANS) contribui para o desenvolvimento das ações de saúde no País, incentivando o desenvolvimento de programas de promoção da saúde e prevenção de risco e doenças, no intuito de modificar o modelo assistencial vigente no sistema de saúde e melhorar a qualidade de vida dos beneficiários de planos de saúde ${ }^{8}$, porém não encontramos até o momento da submissão deste artigo nenhuma referência na literatura que retrate essa iniciativa dos planos de saúde.

Em outro contexto, uma revisão sobre atividade física no ambiente de trabalho afirma que a adaptação desse ambiente para criar oportunidades dos indivíduos serem fisicamente mais ativos pode beneficiar tanto os empregados quanto os empregadores de várias maneiras 9 .

Em resposta ao interesse privado de um plano de saúde e ao interesse público de uma Instituição de obter funcionários mais ativos fisicamente, o AGROS Instituto UFV de Seguridade Social, como plano de saúde, em parceria com a Universidade Federal de Viçosa - Campus Florestal (UFV - CAF) propuseram o Serviço de Atividade Física e Saúde (Espaço Movimento) na cidade de Florestal - MG, com o intuito de atender os servidores ativos e aposentados, também participantes do AGROS, e seus dependentes no plano de saúde.

Dessa forma, o objetivo desse estudo foi avaliar a eficácia desse programa de intervenção com atividade física sobre determinados parâmetros de saúde, tais como, índice de massa corporal (IMC), índice de cintura quadril (ICQ), percentual de gordura corporal (\%GC), percentual de massa magra (\%MM), e o perfil de estado de humor, após seis meses de atividade física regular.

\section{MATERIAL E MÉTODOS}

\section{O Programa - Espaço Movimento}

O Serviço de Atividade Física e Saúde (Espaço Movimento) é uma proposta de continuidade de um estudo piloto, intitulado "Programa integrado de atividade física, esporte e lazer para todos os servidores da UFV Campus Florestal: estudo piloto (PIAFEL-EP)", que foi realizado em convênio entre o AGROS e a UFV CAF no período de julho de 2007 a novembro de 2011.

Só são aptos a participar do Espaço Movimento os servidores da UFV-CAF conveniados ao AGROS ou seus dependentes, que apresentem a liberação médica para a prática de atividade física.

\section{Cuidados éticos}

O estudo foi apreciado e aprovado pelo Comitê de Ética em Pesquisa com Seres Humanos da Universidade Federal de Viçosa, conforme determina a Resolução no 466/12 do Conselho Nacional de Saúde, sob o parecer número 487.635. Todos os indivíduos que ingressam no Espaço Movimento são informados do objetivo do estudo e em concordância assinam o termo de consentimento livre e esclarecido.

\section{Amostra}

Foram selecionados 44 participantes, 17 servidores ativos $(47,7 \pm 10,2$ anos), 10 servidores 
aposentados (66,4 $\pm 3,4$ anos) e 17 dependentes $(43,7 \pm$ 15 anos), sendo 15 mulheres e 29 homens. Fizeram parte da amostra todos os participantes que estavam inseridos há seis meses no Espaço Movimento e que apresentaram uma frequência mínima de seis vezes ao mês.

\section{Avaliações}

No início da intervenção e após seis meses foi realizada uma avaliação física pelo mesmo avaliador, onde foram avaliadas a massa corporal e estatura utilizando uma balança mecânica antropométrica Balmak 111 , com capacidade de até $150 \mathrm{~kg}$ e divisão de $100 \mathrm{~g}$, e até $2 \mathrm{~m}$ com graduação de $0,5 \mathrm{~cm}$ para o cálculo do IMC; fita métrica Cescorf para a medição dos perímetros de cintura e quadril para o cálculo do ICQ; o \%GC e o \%MM (100 - \%GC) segundo o protocolo de dobras cutâneas de Faulkner ${ }^{10}$ utilizando o adipômetro científico Cescorf; e o perfil de estado de humor através da Escala de Humor de Brunel, BRUMS ${ }^{11}$, que se trata de uma escala com 24 itens que se dividem em seis dimensões de humor: tensão, depressão, raiva, vigor, fadiga e confusão.

\section{Treinamento}

Os treinos são preparados pelo profissional de educação física responsável e fundamentados nas recomendações da literatura e nos princípios do treinamento esportivo, destacando que o início e a evolução de cada participante no programa de atividade física são avaliados individualmente, considerando sua prática de atividade física habitual, função física, adaptação aos estímulos e metas estabelecidas, sempre na perspectiva da promoção da saúde e prevenção de riscos e doenças. Os treinamentos acontecem em um ambiente de academia e são compostos por exercícios de alongamentos, aeróbicos e resistidos, os quais são reformulados quando atingido o número total de treinos previstos, podendo variar de um a dois meses, dependendo da frequência semanal do participante.

\section{Tratamento estatístico}

A normalidade da distribuição das variáveis foi verificada por meio do teste Kolmogorov-Smirnov. Para avaliar os resultados das reavaliações dos participantes foi utilizado teste paramétrico ( $\mathrm{t}$ pareado) e não paramétrico (Wilcoxon) para comparação de dois grupos dependentes, conforme o comportamento da variável em questão. O nível de significância foi de p d" 0,05. O programa estatístico utilizado foi o Sigma Stat 3.0.

\section{RESULTADOS}

Os resultados relativos aos dados antropométricos (Tabela 1) apresentam uma diminuição e aumento significativo do \%GC e \%MM, respectivamente, para todos os grupos atendidos. Além disso, o grupo de servidores aposentados apresentou uma diminuição significativa no ICQ $(p=0,032)$.

As respostas à escala de BRUMS (Tabela 2) demonstram uma diminuição significativa da dimensão raiva nos grupos de servidores ativos e aposentados, e um aumento significativo na dimensão vigor no grupo de dependentes.

\section{DISCUSSÃO}

Destacamos como uma limitação do nosso estudo a ausência de um grupo controle, o que nos permitiria atribuir integralmente à prática do exercício físico todos os resultados atingidos, ao mesmo tempo, esse estudo se destaca pela sua grande aplicação prática, pois se trata de dados coletados da realidade de uma proposta de intervenção com atividade física de fluxo contínuo.

Iniciativas que promovam a atividade física devem ser estimuladas nos diferentes ambientes e segmentos, pois parâmetros adequados de saúde estão associados a uma longevidade saudável, ao maior desempenho e satisfação no trabalho, dentre outros aspectos relacionados à qualidade de vida ${ }^{9}$.

Em nosso trabalho os valores antropométricos apresentaram uma diminuição significativa no \%GC após os seis meses de intervenção composta por exercícios de alongamento, aeróbicos e resistidos, assim como no estudo de Barzegari e Amounzad ${ }^{12}$ após oito semanas de treinamento resistido com homens diabéticos tipo 2 . Porém, não houve diferença significativa no atual 
Tabela 1. Comparação dos índices antropométricos antes e pós treinamento (6 meses) dos três grupos atendidos: Servidores Ativos, Servidores Aposentados e Dependentes.

\begin{tabular}{|c|c|c|c|c|c|c|c|c|c|}
\hline & \multicolumn{3}{|c|}{ Servidores Ativos $(n=17)$} & \multicolumn{3}{|c|}{$\begin{array}{l}\text { Servidores Aposentados (n } \\
=10)\end{array}$} & \multicolumn{3}{|c|}{ Dependentes $(n=17)$} \\
\hline & Início & 6 meses & $\mathbf{P}$ & Início & 6 meses & p & Início & 6 meses & p \\
\hline IMC & $\begin{array}{c}27,1 \\
(24,93- \\
31,93) \#\end{array}$ & $\begin{array}{c}27,77 \\
(25,62- \\
30,89)\end{array}$ & 0,678 & $\begin{array}{c}29,13 \pm \\
2,9 ¥\end{array}$ & $\begin{array}{l}28,31 \pm \\
3,04\end{array}$ & 0,068 & $\begin{array}{c}24,12 \\
(23,09- \\
26,42) \#\end{array}$ & $\begin{array}{c}23,88 \\
(22,84- \\
27,44)\end{array}$ & 0,487 \\
\hline ICQ & $\begin{array}{l}0,88 \pm \\
0,06 ¥\end{array}$ & $\begin{array}{c}0,88 \pm \\
0,07\end{array}$ & 0,992 & $\begin{array}{l}0,94 \pm \\
0,07 ¥\end{array}$ & $\begin{array}{c}0,92 \pm \\
0,07\end{array}$ & $\begin{array}{l}0,032 \\
*\end{array}$ & $\begin{array}{c}0,8 \pm 0,08 \\
¥\end{array}$ & $\begin{array}{c}0,79 \pm \\
0,07\end{array}$ & 0,317 \\
\hline$\%$ GC & $\begin{array}{r}22,93 \pm \\
4,73 ¥\end{array}$ & $\begin{array}{c}21,54 \pm \\
4,42\end{array}$ & $\begin{array}{c}0,027 \\
*\end{array}$ & $\begin{array}{l}23,08 \pm \\
4,93 ¥\end{array}$ & $\begin{array}{c}21,39 \pm \\
4,92\end{array}$ & $\begin{array}{c}0,013 \\
*\end{array}$ & $\begin{array}{c}20,77 \pm \\
6,53 ¥\end{array}$ & $\begin{array}{c}19,74 \pm \\
7,1\end{array}$ & $\begin{array}{c}0,034 \\
*\end{array}$ \\
\hline $\begin{array}{c}\% \\
\text { MM } \\
\end{array}$ & $\begin{array}{l}77,07 \pm \\
4,73 ¥ \\
\end{array}$ & $\begin{array}{c}78,5 \pm \\
4,42 \\
\end{array}$ & $\begin{array}{c}0,027 \\
* \\
\end{array}$ & $\begin{array}{l}76,92 \pm \\
4,93 ¥ \\
\end{array}$ & $\begin{array}{c}78,61 \pm \\
4,92 \\
\end{array}$ & $\begin{array}{c}0,013 \\
*\end{array}$ & $\begin{array}{l}79,23 \pm \\
6,53 ¥ \\
\end{array}$ & $\begin{array}{c}80,26 \pm \\
7,1 \\
\end{array}$ & $\begin{array}{c}0,034 \\
*\end{array}$ \\
\hline
\end{tabular}

*Diferença significativa $\mathrm{p}=0,05$.

\# Variável com comportamento não-paramétrico (Wilcoxon) apresentada em valor de mediana e diferença inter-quartil.

$¥$ Variável com comportamento paramétrico (t pareado) apresentada em valor de média e desvio padrão.

\begin{tabular}{|c|c|c|c|c|c|c|c|c|c|}
\hline & \multicolumn{3}{|c|}{ Servidores Ativos $(n=17)$} & \multicolumn{3}{|c|}{$\begin{array}{c}\text { Servidores Aposentados } \\
(\mathrm{n}=\mathbf{1 0})\end{array}$} & \multicolumn{3}{|c|}{ Dependentes $(n=17)$} \\
\hline & Início & $\begin{array}{c}6 \\
\text { meses }\end{array}$ & $\mathbf{P}$ & Início & $\begin{array}{c}6 \\
\text { meses }\end{array}$ & $\mathbf{p}$ & Início & $\begin{array}{c}6 \\
\text { meses }\end{array}$ & p \\
\hline Raiva & $\begin{array}{l}1,06 \pm \\
0,94 ¥\end{array}$ & $\begin{array}{c}0,76 \pm \\
1,08\end{array}$ & $\begin{array}{c}0,044 \\
*\end{array}$ & $\begin{array}{l}0,87 \pm \\
0,93 ¥\end{array}$ & $\begin{array}{c}0,17 \pm \\
0,31\end{array}$ & $\begin{array}{c}0,024 \\
*\end{array}$ & $\begin{array}{l}0,22 \pm \\
0,3 ¥\end{array}$ & $\begin{array}{c}0,32 \pm \\
0,4\end{array}$ & 0,322 \\
\hline Confusão & $\begin{array}{c}0,25(0 \\
-0,75) \\
\#\end{array}$ & $\begin{array}{l}0(0- \\
0,37)\end{array}$ & 0,970 & $\begin{array}{c}0(0- \\
0,25) \#\end{array}$ & $\begin{array}{c}0(0- \\
0)\end{array}$ & 0,5 & $\begin{array}{c}0,5(0- \\
0,5) \#\end{array}$ & $\begin{array}{l}0,25(0 \\
-0,31)\end{array}$ & 0,204 \\
\hline Depressão & $\begin{array}{l}0,5(0- \\
1,56) \#\end{array}$ & $\begin{array}{c}0(0- \\
0,5)\end{array}$ & 0,055 & $\begin{array}{c}0(0- \\
0,25) \#\end{array}$ & $\begin{array}{c}0(0- \\
0)\end{array}$ & 0,25 & $\begin{array}{c}0,25 \\
(0,25- \\
0) \#\end{array}$ & $\begin{array}{c}0(0- \\
0)\end{array}$ & 0,297 \\
\hline Fadiga & $\begin{array}{c}1,25 \\
(0,44- \\
2) \#\end{array}$ & $\begin{array}{c}0,5 \\
(0,19- \\
1,06)\end{array}$ & 0,194 & $\begin{array}{l}0,12(0 \\
-0,5) \#\end{array}$ & $\begin{array}{c}0(0- \\
0)\end{array}$ & 0,438 & $\begin{array}{l}0,88 \pm \\
0,88 ¥\end{array}$ & $\begin{array}{c}0,56 \pm \\
0,5\end{array}$ & 0,113 \\
\hline Tensão & $\begin{array}{l}1,47 \pm \\
0,75 ¥\end{array}$ & $\begin{array}{c}1,12 \pm \\
0,93\end{array}$ & 0,072 & $\begin{array}{l}1,27 \pm \\
0,83 ¥\end{array}$ & $\begin{array}{c}0,8 \pm \\
0,48\end{array}$ & 0,073 & $\begin{array}{c}1 \pm \\
0,64 ¥\end{array}$ & $\begin{array}{c}1,01 \pm \\
0,63\end{array}$ & 0,944 \\
\hline Vigor & $\begin{array}{c}2,5 \pm \\
0,85 ¥\end{array}$ & $\begin{array}{c}2,73 \pm \\
0,64\end{array}$ & 0,328 & $\begin{array}{l}3,02 \pm \\
0,56 ¥\end{array}$ & $\begin{array}{c}3 \pm \\
0,72\end{array}$ & 0,918 & $\begin{array}{l}2,21 \pm \\
0,97 ¥\end{array}$ & $\begin{array}{c}2,96 \pm \\
0,55\end{array}$ & $\begin{array}{c}0,003 \\
*\end{array}$ \\
\hline
\end{tabular}

*Diferença significativa $\mathrm{p}=0,05$.

\# Variável com comportamento não-paramétrico (Wilcoxon) apresentada em valor de mediana e diferença inter-quartil.

¥ Variável com comportamento paramétrico (t pareado) apresentada em valor de média e desvio padrão.

estudo no IMC dos participantes, o que foi observado no estudo de Barzegari e Amounzad ${ }^{12}$.

O IMC dos avaliados provavelmente não foi alterado devido ao aumento significativo correspondente do $\% \mathrm{MM}$, o que se torna um resultado importante principalmente para o grupo de servidores aposentados, com idade média de 66,4 anos, pois devido ao processo de envelhecimento há uma expressiva perda de massa magra ${ }^{13,14}$, chegando a reduzir a força muscular em 1,5\% ao ano entre 50 e 60 anos de idade e em até $3 \%$ acima dessa idade ${ }^{15}$.

O estudo de Kennis et al..$^{16}$ apresenta melhoras significativas em força em um grupo de idosos submetidos a um ano de treinamento resistido, porém, 
uma vez que a intervenção foi interrompida este não foi capaz de atenuar o declínio nas características de força relacionadas à idade, quando mensurado sete anos após o término da intervenção. Esses resultados reforçam a importância da proposta de intervenção do Espaço Movimento que é contínua, e não prevê interrupção ao treinamento, a menos que o participante deseje abandonar o Programa.

$\mathrm{O}$ estudo de Venturim e $\mathrm{Cade}^{17}$ com mulheres obesas (IMC > 30) que apresenta uma intervenção semelhante à do nosso estudo no que diz respeito à composição do treinamento físico, foi observado uma diminuição significativa no IMC, \%GC e no ICQ após 4 meses de intervenção. No estudo atual, além da redução e aumento significativo do \%GC e \%MM, respectivamente, houve uma redução significativa no ICQ $(p=0,032)$ para o grupo de servidores aposentados.

Embora o ICQ não tenha apresentado diferença significativa para todos os grupos, esse é um índice extremamente importante e de fácil mensuração para estimar o risco de eventos cardiovasculares, tendo em vista que a gordura central está diretamente associada a esses ${ }^{18}$, além de seu valor aumentado ser um fator de risco para a mortalidade tota ${ }^{19}$.

Dessa forma, principalmente para o grupo de servidores aposentados, que de modo geral não possui compromissos laborativos, e por ser um grupo que apresenta baixa aderência e permanência na prática regular de exercícios físicos ${ }^{20}$, os resultados apresentados aqui assumem grande importância no aspecto preventivo.

O nível de estado de humor tem sido muito utilizado para avaliar os efeitos psicológicos do exercício físico ${ }^{21-23}$, principalmente para abranger o amplo conceito de saúde definido pela OMS e por este ter se tornado uma alternativa não-farmacológica, econômica, saudável e de maior adesão na prevenção e tratamento de distúrbios psicológicos e na promoção da saúde mental $^{24}$.

O estudo de Brandt et al. ${ }^{25}$ propôs mensurar o nível de estado de humor de participantes de um programa semelhante ao desse estudo que atendem pessoas com doenças cardiovasculares e/ou metabólicas por meio da prática de exercícios físicos, no qual também foi utilizado o mesmo instrumento de avaliação, a escala de BRUMS ${ }^{11}$, que é uma versão reduzida do Profile of Mood States (POMS).

Porém, no estudo citado acima foi realizado um único momento de avaliação, a partir do qual foi possível sugerir que os estados de humor positivos podem estar relacionados ao programa de exercício físico que os indivíduos estão envolvidos. O que é reforçado pelos resultados encontrados no presente estudo, diante de uma redução significativa na dimensão raiva para o grupo dos servidores ativos $(\mathrm{p}=0,044)$ e aposentados $(\mathrm{p}=0,024)$, e um aumento significativo na dimensão vigor $(p=0,033)$ no grupo de dependentes após seis meses de exercício físico regular.

Um comportamento semelhante foi observado no estudo de Oliveira et $a l .{ }^{26}$ que avaliou o efeito de uma proposta de intervenção com atividade física com os dependentes dos funcionários de uma determinada empresa, no qual foi apresentado um aumento significativo na dimensão vigor e uma redução significativa na dimensão tensão após seis meses. No nosso estudo o grupo de dependentes também apresentou um aumento significativo na dimensão vigor $(p=0,033)$. Esses resultados reforçam o posicionamento de Mello et al. ${ }^{27}$ de que o exercício físico sistematizado causa diversos benefícios nas esferas física e mental do ser humano, proporcionando uma maior qualidade de vida.

$\mathrm{Na}$ literatura há muitos estudos sobre intervenção com atividade física na saúde pública ${ }^{28}$, outros dentro de empresas privadas ${ }^{29}$ mas esse é pioneiro em avaliar essa intervenção no domínio de um plano de saúde.

\section{CONCLUSÃO}

Os resultados sugerem que o objetivo do Espaço Movimento tem sido atingido, dado a melhora nos parâmetros avaliados. Além disso, os dados apresentados nesse estudo servem de incentivo para empresas que se preocupam com a qualidade de vida dos seus funcionários, assim como para outros planos de saúde que optem por gastar com prevenção ao invés de tratamento para o desenvolvimento de propostas semelhantes a essa.

\section{AGRADECIMENTOS}

Agradecemos aos estudantes do curso de Educação Física da Universidade Federal de Viçosa Campus Florestal, Michele Aparecida Almeida e Edson Pablo de Jesus Gomes, pela valiosa contribuição no processo de intervenção, e a todos os demais membros da equipe multiprofissional do Espaço Movimento. 


\section{REFERÊNCIAS}

1. Lopes MSV, Saraiva KRO, Fernandes AFC, Ximenes LB. Análise do conceito de promoção da saúde. Texto Contexto - Enferm. 2010; 19 (3): 461-8.

2. WHO (World Health Organization). Constitution of the World Health Organization. Basic Documents. WHO. Genebra. 1946.

3. Pozena R, Cunha NFS. Projeto "construindo um futuro saudável através da prática da atividade física diária". Saúde Soc. 2009; 18 (Supl 1): 52-6.

4. Lee I-Min, Shiroma EJ, Lobelo F, Puska P, Blair SN, Katzmarzyk PT. Effect of physical inactivity on major non-communicable diseases worldwide: an analysis of burden of disease and life expectancy. Lancet. 2012; 380 (9838): 219-29.

5. Brito AKA, Silva FIC, França NM. Programas de intervenção nas escolas brasileiras: uma contribuição da escola para a educação em saúde. Saúde debate. 2012; 36 (95): 624-32.

6. Brasil. Ministério da Saúde. Secretaria de Vigilância em Saúde. Política nacional de promoção da saúde / Ministério da Saúde, Secretaria de Atenção à Saúde. Brasília: Ministério da Saúde, 2006.

7. Malta DC, Silva JB. Policies to promote activity in Brazil. Lancet. 2012; 380 (9838): 195-6.

8. Agência Nacional de Saúde Suplementar (Brasil) Promoção da Saúde e Prevenção de Riscos e Doenças na Saúde Suplementar: manual técnico. $3^{a}$ Edição. Rio de Janeiro: ANS; 2007.

9. Curry R. Investing in Physical Activity Through in Workplace: A Toolkit for Policy Development. Hamilton: Public Health Services, 2012.

10. Faulkner JA. Physiolgy of swimming and diving. In: $\mathrm{H}$. FALLS. Exercise Physiology, Baltimore: Academic Press, 1968.

11. Terry PC, Lane AM, Fogarty GJ. Construct validity of the Profile of Mood States - Adolescents for use with adults. Psychol Sport Exerc. 2003; 4: 125-39.

12. Barzegari A, Amouzad MH. Effects of 8 weeks resistance training on plasma vaspin and lipid profile levels in adult men with type 2 diabetes. Caspian $\mathrm{J}$ Intern Med. 2014; 5(2): 103-8.

13. Silva TAA, Junior AF, Pinheiro MM, Szejnfeld VL. Sarcopenia associada ao envelhecimento: aspectos etiológicos e opções terapêuticos. Rev Bras Reumatol. 2006; 46 (6): 391-7.

14. Lacourt MX, Marini LL. Decréscimo da função muscular decorrente do envelhecimento e a influência na qualidade de vida do idoso: uma revisão de literatura. Rev Bras de Ciên do Envelh Hum. 2006; 3 (1)114-21.

15. von Haehling S, Morley JE, Anker SD. An overview of sarcopenia: facts and numbers on prevalence and clinical impact. J Cachexia Sarcopenia Muscle. 2010; 1: 129-33.

16. Kennis E, Verschueren SM, An Bogaerts, Roie EV, Boonen S, Celecluse C. Long-Term Impact of Strength Training on Muscle Strength Characteristics in Older Adults. Arch Phys Med Rehabil. 2013; 94(11): 2054-60.

17. Venturim LMVP, Cade NV. Efeitos do programa "P.E.S.O" (Promoção de estilo de vida saudável na obesidade) sobre variáveis antropométricas, hemodinâmicas e bioquímicas. Rev Bras Ativ Fís Saúde. 2007; 12(1): 1926.
18. Picon PX, Leitão CB, Gerchman F, Azevedo MJ, Silveiro SP, Gross JL, Canani LH. Medida da cintura e razão cintura/quadril e identificação de situações de risco cardiovascular: estudo multicêntrico em pacientes com Diabetes Melito tipo 2. Arq Bras Endocrinol Metab. 2007; 51(3): 443-9.

19. Cabrera MAS, Wajngarten M, Gebara OCE, Diament J. Relação do índice de massa corporal, da relação cintura-quadril e da circunferência abdominal com a mortalidade em mulheres idosas: seguimento de 5 anos. Cad Saúde Pública. 2005; 21 (3): 767-75.

20. Freitas CMSM, Santiago MS, Viana AT, Leão AC, Freyre C. Aspectos motivacionais que influenciam a adesão e manutenção de idosos a programas de exercícios físicos. Rev Bras Cineantropom Desempenho Hum. 2007; 9 (1): 92-100.

21. Song $C$, Joung $D$, Ikei $H$, Igarashi M, Aga M, Park BumJin, et al. Physiological and psychological effects of walking on young males in urban parks in winter. $J$ Physiol Anthropol. 2013; 32 (18):1-5.

22. Ariza-garcía A, Galiano-Castillo N, Cantarero-Villanueva I, Fernández-Lao C, Díaz-Rodríguez L, Arroyo-Morales M. Influence of physical inactivity in psychophysiolocigal state of breast cancer survivors. Eur J Cancer Care. 2013; 22(6): 738-745.

23. Steffens RAK, Liz CM, Viana MS, Brandt R, Oliveira LMA, Andrade A. Praticar caminhada melhora a qualidade do sono e os estados de humor em mulheres com síndrome da fibromyalgia. Rev Dor. 2011; 12 (4): 327-31.

24. Werneck FZ, Navarro CA. Nível de atividade física e estado de humor em adolescentes. Psic: Teor e Pesq. 2011; 27 (2): 189-93.

25. Brandt R, Viana MS, Steffens RAK, Silva RB, Sties SW, Gonzáles Al, et al. Perfil de humor e fatores associados em participantes de um programa de reabilitação cardiopulmonar e metabólica. J Bras Psiquiatr. 2012; 61 (2): $72-7$.

26. Oliveira TFB, Laterza MC, Ferreira R, Werneck FZ, Paixão JA, Coelho EF. Efetividade de um programa de avaliação e prescrição de exercícios físicos para mulheres. Rev Bras Ciênc Saúde. 2011; 9 (30): 1-8.

27. Mello MT, Boscolo RA, Esteves AM, Tufik S. O exercício físico e os aspectos psicobiológicos. Rev Bras Med Esporte. 2005; 11 (3): 203-7.

28. Loch MR, Brunetto BC, Rodrigues CG, Nahas MV. A Saúde Pública nos anais do Congresso Brasileiro de Atividade Física e Saúde (1997-2009): revisão sistemática. Rev Bras Ativ Fís Saúde. 2011; 16 (2): 162167.

29. Reis A, Mansini G, Leite F. Instituto de Estudos de Saúde Suplementar. Promoção de saúde nas empresas: casos de sucesso. Disponível em:<http://www.iess.org.br/ promosaudecasosucessoiess.pdf $>$. Acesso em: $28 \mathrm{de}$ abril de 2016.

Correspondência

Nome completo: Valéria Cristina de Faria

Rua José de Souza Maciel, 192. Bairro Dona Suzana.

Florestal - Minas Gerais - Brasil - CEP: 35.690-000

E-mail: valeriaefiufv@yahoo.com.br 